\title{
Liver Transplantation for Intermediate-Stage Hepatocellular Carcinoma
}

\author{
Naoko Kamo Toshimi Kaido Shintaro Yagi Hideaki Okajima \\ Shinji Uemoto
}

Division of Hepato-Biliary-Pancreatic and Transplant Surgery, Department of Surgery,

Graduate School of Medicine, Kyoto University, Kyoto, Japan

\section{Keywords}

Intermediate-stage hepatocellular carcinoma - Kyoto criteria · Pretreatment - Transarterial chemoembolization · Transplantation

\begin{abstract}
Transarterial chemoembolization is the standard treatment for patients with intermediatestage hepatocellular carcinoma (HCC) according to the Barcelona Clinic Liver Cancer staging system. However, in Japan, not a few patients with intermediate-stage HCC undergo liver transplantation (LT). The present study investigated characteristics and outcomes of LT for intermediate-stage HCC. Between February 1999 and November 2016, a total of 226 patients underwent LT for HCC at our institute. Among these, 56 patients showed intermediate-stage HCC (24.8\%). We examined overall survival and recurrence rate after LT according to our extended criteria (maximum size $\leq 5 \mathrm{~cm}$, number $\leq 10$, des-gamma-carboxy prothrombin $\leq 400$ $\mathrm{mAU} / \mathrm{mL}$ ) and pretreatment. One-, 3-, and 5-year overall survival and recurrence rates of LT for intermediate-stage HCC were $88 / 64 / 58 \%$ and $22 / 34 / 44 \%$, respectively. One-, $3-$, and 5 -year overall survival and recurrence rates in patients within $(n=35)$ the criteria $(94 / 80 / 80 \%$ and $9 / 15 / 22 \%$, respectively) were significantly better than those in patients beyond $(n=21)$ the criteria $(81 / 43 / 29 \%, p=0.002$ and $39 / 41 / 66 \%, p=0.001$, respectively). Forty-nine cases $(88 \%)$ had a history of pretreatment. In patients within our extended criteria, overall survival and recurrence rates did not differ significantly between patients with $(n=31)$ and without $(n=4)$ pretreatment. In conclusion, outcomes after LT for intermediate-stage HCC are more favorable if patients meet our extended criteria.




\section{Liver Cancer}

\section{Introduction}

Hepatocellular carcinoma (HCC) is the most common type of primary liver cancer in adults with increasing incidence and mortality rates $[1,2]$. Although most cases occur in Asia and Africa, the incidence has also been rising in developed countries. Regarding mortality, HCC is the second leading cause of cancer-related death in the world [2]. In the last two decades, multidisciplinary treatments for HCC have improved, including hepatic resection (HR), radiofrequency ablation (RFA), transarterial chemoembolization (TACE), and liver transplantation (LT). Selection of a particular therapy depends on the tumor features, degree of underlying liver function, and availability of the treatment modality and local clinical expertise [3-5].

The Barcelona Clinic Liver Cancer (BCLC) classification, the most widely applied staging system, divides HCC patients into 5 stages based on tumor stage, cirrhosis stage, and performance status, and proposes a treatment algorithm that is associated with a different prognosis [6]. Intermediate-stage HCC, defined as BCLC stage B, has the following host variables: multinodular HCC without vascular invasion or distant metastasis, Child-Pugh class A or B, and a performance status of 0 . In the BCLC guidelines, TACE is commonly recommended for the treatment of intermediate-stage HCC. However, intermediate-stage HCC includes a wide variety of HCCs and patients with intermediate-stage HCC undergo various kinds of treatment, including local therapy, TACE, and LT in the clinical setting.

LT now plays an important role in the treatment of patients with HCC worldwide. The Japanese treatment algorithm for HCC recommends LT for patients with Child-Pugh class C liver function who are within the Milan criteria (MC) [7]. However, expanded transplantation criteria for HCC beyond the MC, especially in living-donor LT (LDLT), have been applied by many transplant centers [8-13]. Clinically, patients with Child-Pugh class A or B liver function who cannot undergo HR, RFA, or TACE because of liver dysfunction or tumor location sometimes receive LDLT. However, little is known about the characteristics and outcomes of LDLT for intermediate-stage HCC.

This retrospective study analyzed the impact of LDLT for intermediate-stage HCC on long-term survival and recurrence, with a particular focus on our expanded criteria and pretreatment.

\section{Patients and Methods}

A total of 226 patients underwent adult LDLT for HCC at Kyoto University Hospital between February 1999 and November 2016. Among these, 56 patients with intermediate-stage HCC were enrolled in this retrospective study. The database was designed for patients with intermediate-stage HCC. This study was approved by the ethics committee at Kyoto University and conducted in accordance with the Declaration of Helsinki of 2000.

Standard selection criteria for LT recipients and the surgical techniques for both donor and recipient operations have been described in detail [14-16]. We preoperatively estimated graft and remnant liver volumes in donors using 3-dimensional reconstructed images of the hepatic vascular anatomy produced using a software package based on reconstructed, multi-detector row computed tomography of the liver. Our selection criteria for LDLT for HCC until December 2006 included HCCs without any restriction of size or tumor number and without gross vascular involvement or distant metastasis at the time of preoperative imaging. Since January 2007, we have applied the Kyoto criteria (KC) on the basis of risk factor analysis [13]. The KC consist of three independent significant risk factors for recurrence: tumor number $\leq 10$, maximal diameter of each tumor $\leq 5 \mathrm{~cm}$, and serum des-gamma-carboxy prothrombin concentration $\leq 400 \mathrm{mAU} / \mathrm{mL}$.

Until January 2011, we applied the standard immunosuppression protocol consisting of tacrolimus and low-dose steroid $[17,18]$. After February 2011, we used a steroid-free protocol with mycophenolate mofetil, as described elsewhere, with the exception of patients with ABO-incompatible transplants or with steroid administration before LT [19]. No patients were switched to mammalian target of rapamycin inhibitor. 


\section{Liver Cancer}

Table 1. Clinicopathological characteristics of patients $(n=56)$

\begin{tabular}{l|l}
\hline \multicolumn{2}{l}{ Liver Cancer } \\
\hline DOI: $10.1159 / 000487058$ & $\begin{array}{l}\text { C } 2018 \text { S. Karger AG, Basel } \\
\text { www.karger.com/lic }\end{array}$ \\
\hline
\end{tabular}

Kamo et al.: LT for Intermediate-Stage HCC

\begin{tabular}{lc}
\hline Age, years & $55(22-69)$ \\
Sex (male/female) & $43 / 13$ \\
Etiology & \\
$\quad$ Viral hepatitis C & 28 \\
$\quad$ Viral hepatitis B & 18 \\
$\quad$ Viral hepatitis C/B & 2 \\
$\quad$ Others & 8 \\
AFP, ng/mL & $100(2-212,220)$ \\
DCP, mAU/mL & $100(2-20,600)$ \\
Child-Pugh (A/B/C) & $24 / 32 / 0$ \\
MELD & $11(4-22)$ \\
Maximum tumor size, cm & $3.4(1-26)$ \\
Tumors, $n$ & $6(2-186)$ \\
Classification (well/mod./poor) & $4 / 39 / 13$ \\
Microvascular invasion & $31(55 \%)$ \\
Milan criteria met & $0(0 \%)$ \\
Kyoto criteria met & $35(63 \%)$
\end{tabular}

AFP, $\alpha$-fetoprotein; DCP, des-gamma-carboxy prothrombin; MELD, model for end-stage liver disease.

Table 2. Clinicopathological characteristics of patients according to the KC

\begin{tabular}{llcc}
\hline Characteristics & Within KC $(n=35)$ & Beyond KC $(n=21)$ & $p$ value \\
\hline Age, years & $57(22-69)$ & $55(23-62)$ & 0.38 \\
Sex (male/female) & $29 / 6$ & $14 / 7$ & 0.16 \\
Etiology & & & 0.96 \\
$\quad$ Viral hepatitis C & 17 & 11 & \\
$\quad$ Viral hepatitis B & 12 & 6 & 0.28 \\
$\quad$ Viral hepatitis C/B & 1 & 1 & 0.01 \\
$\quad$ Others & $51(2-3,564)$ & $237(3-212,220)$ & 1.00 \\
AFP, ng/mL & $54(2-400)$ & $443(12-20,600)$ & 0.40 \\
DCP, mAU/mL & $15 / 20 / 0$ & $9 / 12 / 0$ & 0.14 \\
Child-Pugh (A/B/C) & $11(6-22)$ & $10(4-17)$ & 0.03 \\
MELD & $3.4(1-5)$ & $3.6(1-26)$ & 0.26 \\
Maximum tumor size, cm & $5(2-10)$ & $12(2-186)$ & 0.06 \\
Tumors, $n$ & $1 / 26 / 8$ & $3 / 13 / 5$ & $15(71 \%)$ \\
Classification (well/mod./poor) & $16(46 \%)$ & & \\
Microvascular invasion & & & \\
\hline
\end{tabular}

AFP, $\alpha$-fetoprotein; DCP, des-gamma-carboxy prothrombin; MELD, model for end-stage liver disease.

Age, sex, tumor size, number, $\alpha$-fetoprotein, des-gamma-carboxy prothrombin, pathological classification, etiology, and model for end-stage liver disease score for each patient were analyzed. Overall survival and recurrence rates after LT for all cases, within and beyond the $\mathrm{KC}$, and with or without treatment including TACE, RFA, and HR before LT, were also analyzed.

\section{Statistical Analysis}

Consecutive variables are expressed as medians and ranges. Patient characteristics between groups were compared using the $\chi^{2}$ test for multinomial categorical variables and the paired $t$ test for consecutive variables. Cumulative overall survival and recurrence rates were calculated using the Kaplan-Meier method, and differences between curves were evaluated using the log-rank test. We considered values of $p<0.05$ as significant. All data were statistically analyzed using JMP pro version 12 software (SAS Institute, Cary, NC, USA). 


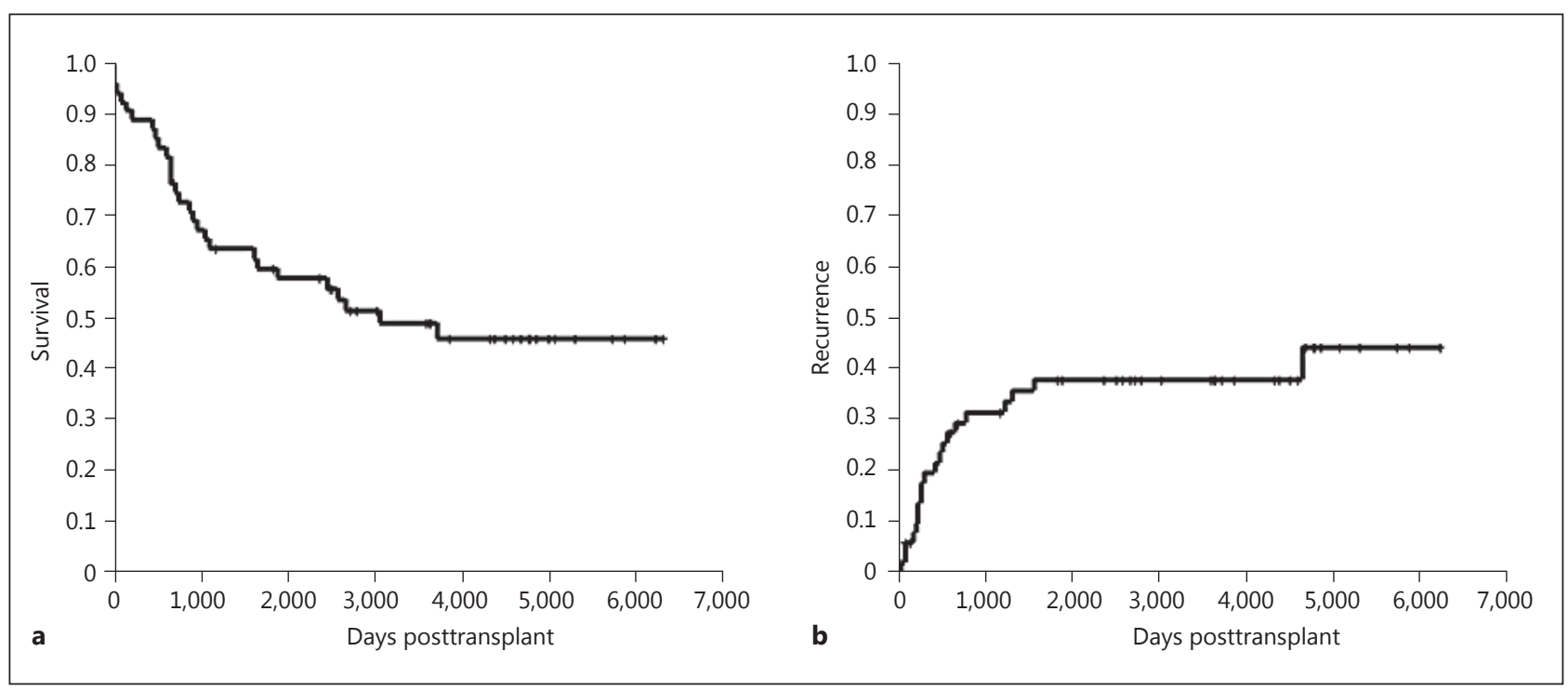

Fig. 1. Overall survival (a) and recurrence (b) rates of LT for intermediate-stage HCC. HCC, hepatocellular carcinoma; LT, liver transplantation.

\section{Results}

Clinicopathological characteristics of patients with intermediate-stage HCC are shown in Table 1. Median age was 55 years (range, 22-69). Forty-three patients were male (77\%). No patients were categorized as Child-Pugh class $\mathrm{C}$, and the proportion of those with ChildPugh classes A and B was 43 and 57\%, respectively. No patients were within the MC. Thirtyfive recipients were beyond the $\mathrm{MC}$ and within the $\mathrm{KC}$, and 21 patients were beyond the $\mathrm{KC}$. Table 2 shows the clinicopathological characteristics of patients according to the KC.

One-, 3-, and 5-year overall survival rates were 88, 64, and 58\%, respectively (Fig. 1a). One-, 3-, and 5-year recurrence rates were 22 , 34, and 44\%, respectively (Fig. 1b).

Next, we analyzed outcomes in terms of the KC. The 1-, 3-, and 5-year overall survival rates in patients within our criteria $(n=35)(94 / 80 / 80 \%)$ were significantly higher than those for patients beyond the KC $(n=21)(81 / 43 / 29 \% ; p=0.002)$ (Fig. 2a). On the other hand, $1-, 3-$, and 5-year recurrence rates in patients within the KC $(9 / 15 / 22 \%)$ were significantly lower than those for patients beyond the KC (39/41/66\%; $p=0.001)$ (Fig. 2b).

Forty-nine cases (88\%) had a history of pretreatment (Fig. 3). Forty-three cases had TACE, 30 cases had RFA, 12 cases HR, and 5 cases all treatments. Table 3 shows the clinicopathological characteristics of patients with or without pretreatment. All Child-Pugh class A patients had a history of pretreatment. Furthermore, Child-Pugh class A patients with pretreatment were significantly more frequent than those without pretreatment. Overall survival rates tended to be lower in patients with pretreatment $(n=49)$ than in those without pretreatment $(n=7)$, although these differences were not significant ( $p=0.087$; Fig. 4a). Recurrence rates did not differ significantly between the 2 groups ( $p=0.175$; Fig. 4 b).

Next, in patients within the $\mathrm{KC}$, the 1-, 3-, and 5-year overall survival rates in groups with $(n=31)$ or without $(n=4)$ pretreatment were 93,77 , and $77 \%$ versus 100,100 , and $100 \%$, respectively, and survival rates did not differ significantly between patients with or without pretreatment ( $p=0.198$; Fig. 5a). Similarly, 1-, 3-, and 5-year recurrence rates in groups with or without pretreatment were 10,17 , and $21 \%$ versus 0,0 , and $0 \%$, respectively. Recurrence rates did not differ significantly between the 2 groups ( $p=0.288$; Fig. $5 b$ ). 
Kamo et al.: LT for Intermediate-Stage HCC

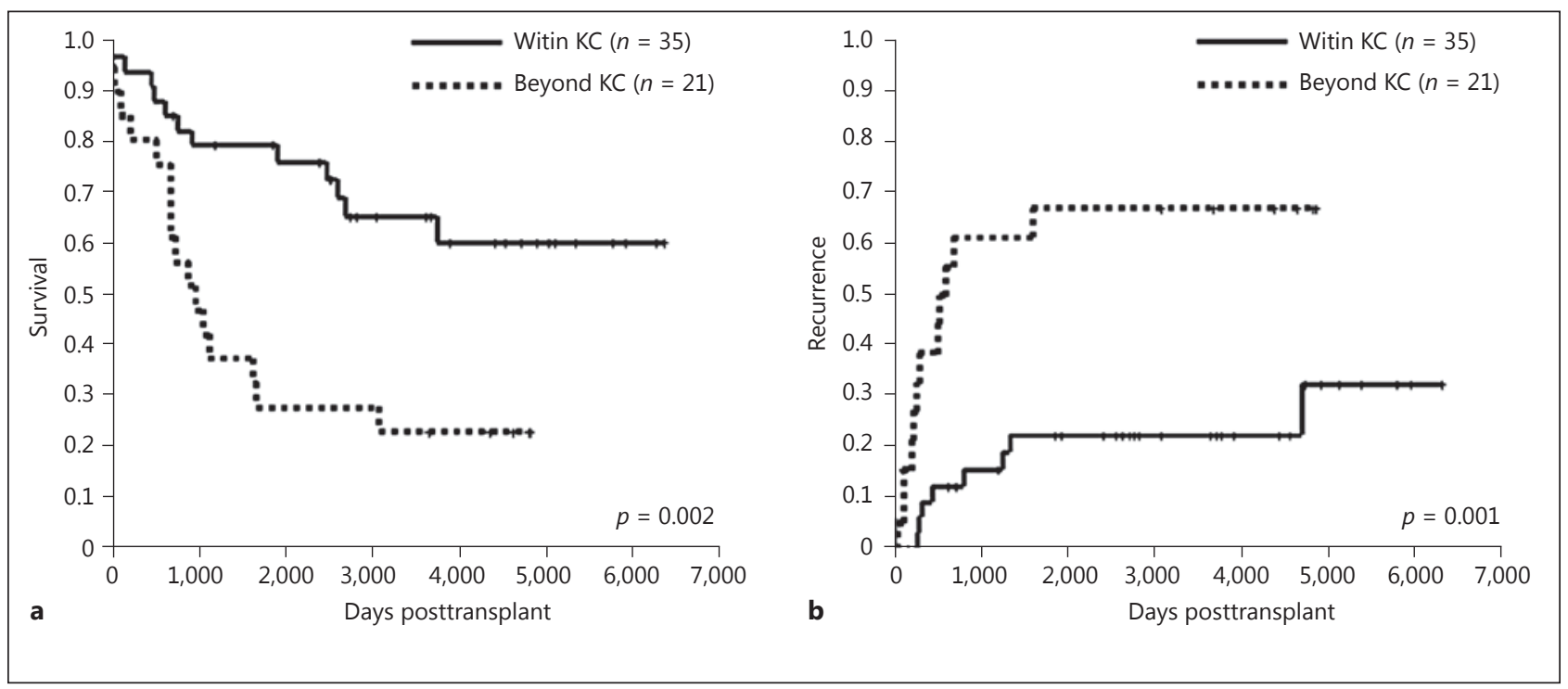

Fig. 2. Overall survival (a) and recurrence (b) rates of LT for intermediate-stage HCC within and beyond the KC. HCC, hepatocellular carcinoma; KC, Kyoto criteria; LT, liver transplantation.

Fig. 3. Pretreatment for intermediate-stage HCC. HCC, hepatocellular carcinoma; TACE, transcatheter arterial chemoembolization; RFA, radiofrequency ablation.

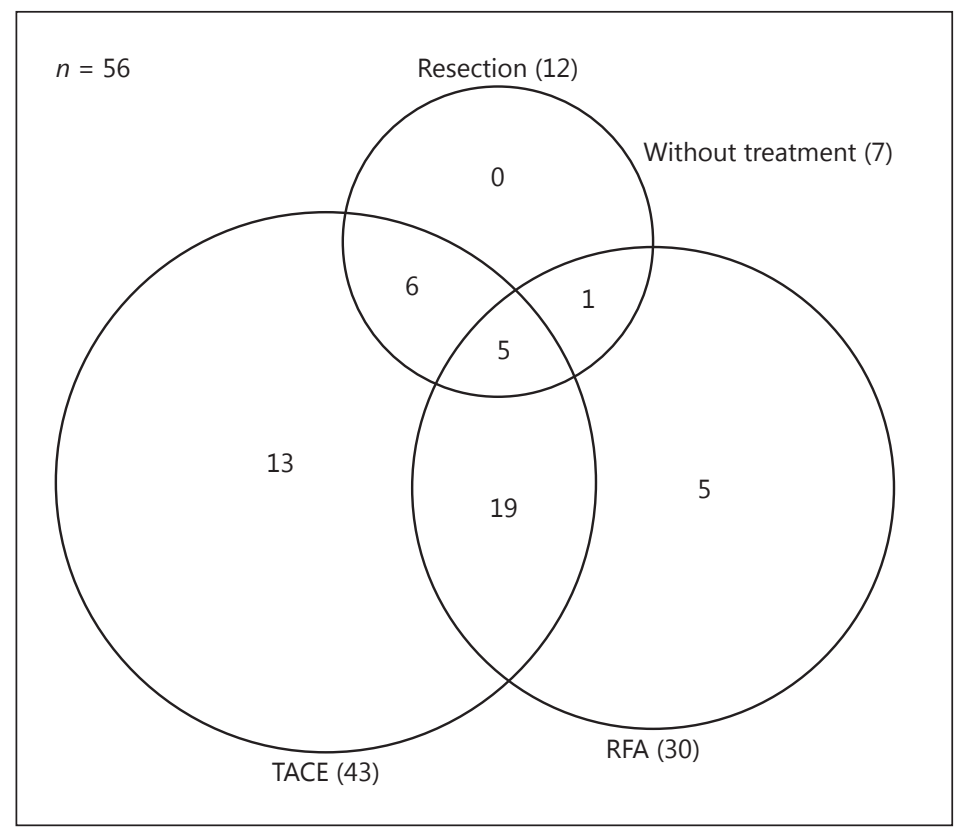

Table 4 shows the clinicopathological characteristics of patients with pretreatment according to the KC. In patients with pretreatment, the 1-, 3-, and 5-year overall survival rates in groups within $(n=31)$ or beyond $(n=18)$ the KC were 93,77 , and $77 \%$ versus 81,43 , and $29 \%$, respectively, and there were significant differences between patients within and beyond the KC ( $p=0.002$; Fig. 6a). Similarly, 1-, 3-, and 5-year recurrence rates in groups within or beyond the $\mathrm{KC}$ were 10,17 , and $21 \%$ versus 38,61 , and $66 \%$, respectively. Recurrence rates also differed significantly between the 2 groups ( $p=0.001$; Fig. $6 \mathrm{~b}$ ). 


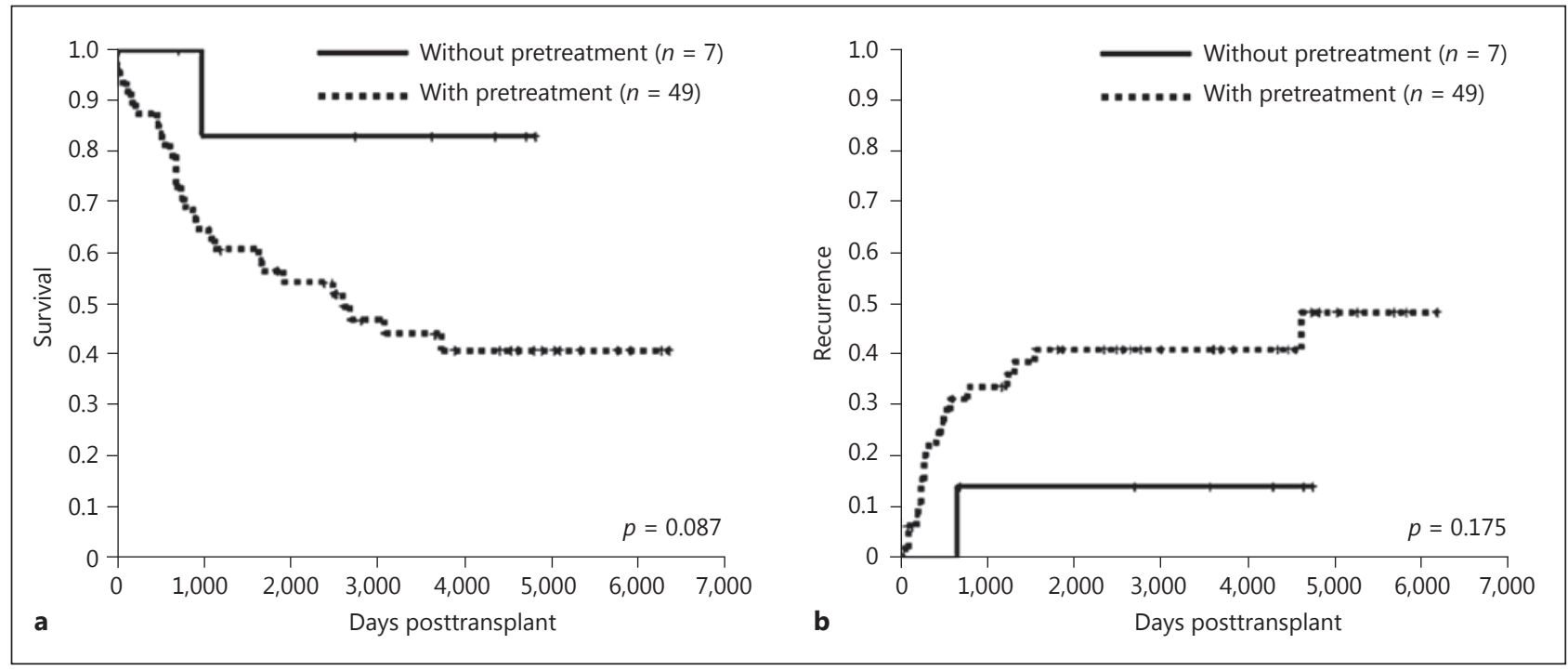

Fig. 4. Overall survival (a) and recurrence (b) rates of LT for intermediate-stage HCC with or without pretreatment. HCC, hepatocellular carcinoma; LT, liver transplantation.

Table 3. Clinicopathological characteristics of patients with or without pretreatment

\begin{tabular}{lccc}
\hline Characteristics & $\begin{array}{l}\text { With pretreatment } \\
(n=49)\end{array}$ & $\begin{array}{l}\text { Without pretreatment } \\
(n=7)\end{array}$ & $p$ value \\
\hline Age, years & $55(22-69)$ & $55(30-64)$ & 0.98 \\
Sex (male/female) & $37 / 12$ & $6 / 1$ & 0.55 \\
Etiology & 25 & 3 & 0.88 \\
$\quad$ Viral hepatitis C & 15 & 3 & 0.68 \\
$\quad$ Viral hepatitis B & 2 & 0 & 0.56 \\
$\quad$ Viral hepatitis C/B & 7 & 1 & 0.01 \\
$\quad$ Others & $93.9(2-212,220)$ & $107(23.8-2,093)$ & 0.28 \\
AFP, ng/mL & $100(2-20,600)$ & $107(12-9,470)$ & 0.62 \\
DCP, mAU/mL & $24 / 25 / 0$ & $0 / 7 / 0$ & 0.29 \\
Child-Pugh (A/B/C) & $10(4-22)$ & $13(10-16)$ & 0.23 \\
MELD & $3.3(1-26)$ & $4.8(3-7)$ & 0.11 \\
Maximum tumor size, cm & $6(2-186)$ & $3(2-4)$ & - \\
Tumors, $n$ & $3 / 32 / 14$ & $1 / 6 / 0$ & 0.76 \\
Classification (well/mod./poor) & $28(57 \%)$ & $3(43 \%)$ & $0(0 \%)$ \\
Microvascular invasion & $0(0 \%)$ & $4(57 \%)$ & \\
Milan criteria met & $31(63 \%)$ & & \\
Kyoto criteria met & & & \\
\hline
\end{tabular}

AFP, $\alpha$-fetoprotein; DCP, des-gamma-carboxy prothrombin; MELD, model for end-stage liver disease.

\section{Discussion}

In this retrospective study, we first clarified long-term outcomes after LDLT for intermediate-stage HCC. Five-year overall survival and recurrence rate for all cases overall were not satisfactory (58 and 44\%, respectively). However, among patients within our extended criteria, the 5-year overall survival (80\%) and recurrence rate $(22 \%)$ after LT were signifi- 


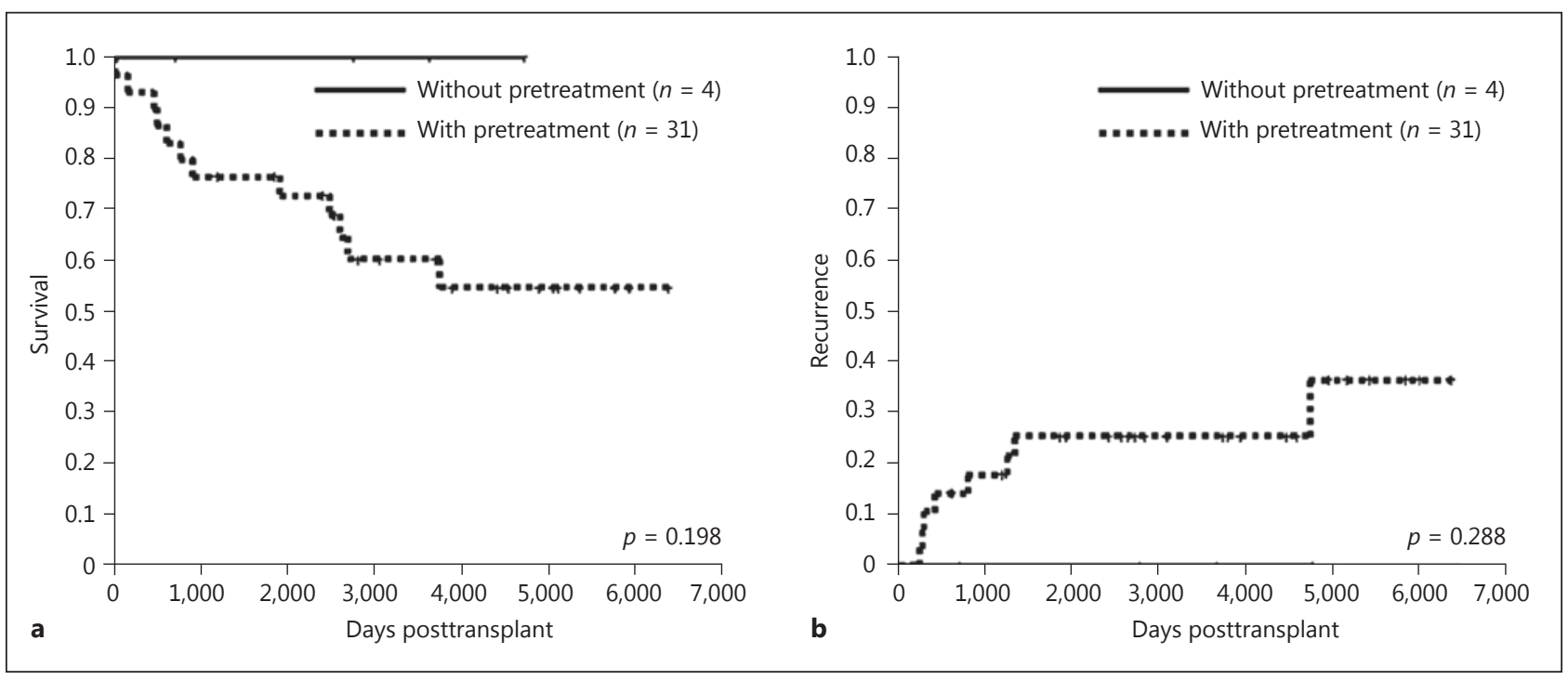

Fig. 5. Overall survival (a) and recurrence (b) rates of LT for intermediate-stage HCC within KC with or without pretreatment. HCC, hepatocellular carcinoma; KC, Kyoto criteria; LT, liver transplantation.

Table 4. Clinicopathological characteristics of patients with pretreatment according to the KC

\begin{tabular}{llcc}
\hline Characteristics & Within KC $(n=31)$ & Beyond KC $(n=18)$ & $p$ value \\
\hline Age, years & $57(22-69)$ & $55(23-62)$ & 0.65 \\
Sex (male/female) & $25 / 6$ & $12 / 6$ & 0.27 \\
Etiology & & & 0.91 \\
$\quad$ Viral hepatitis C & 15 & 10 & \\
$\quad$ Viral hepatitis B & 10 & 5 & \\
$\quad$ Viral hepatitis C/B & 1 & 1 & \\
$\quad$ Others & 5 & 2 & 0.28 \\
AFP, ng/mL & $51(2-3,564)$ & $249(3-212,220)$ & 0.04 \\
DCP, mAU/mL & $56(2-400)$ & $374(12-20,600)$ & 0.91 \\
Child-Pugh (A/B/C) & $15 / 16 / 0$ & $9 / 9 / 0$ & 0.37 \\
MELD & $11(6-22)$ & $10(4-17)$ & 0.23 \\
Maximum tumor size, cm & $3.3(1-5)$ & $16(1-26)$ & 0.03 \\
Tumors, $n$ & $5(2-10)$ & $3 / 9 / 6$ & 0.03 \\
Classification (well/mod./poor) & $0 / 23 / 8$ & $13(72 \%)$ & 0.10 \\
Microvascular invasion & $15(48 \%)$ & & \\
\end{tabular}

AFP, $\alpha$-fetoprotein; DCP, des-gamma-carboxy prothrombin; MELD, model for end-stage liver disease.

cantly better than those for patients beyond the criteria (29 and 66\%, respectively), in line with our previous report showing favorable long-term outcomes in patients within the KC [20]. These findings show that the $\mathrm{KC}$ are useful even in the subgroup of patients with intermediate-stage HCC.

In the present study, most cases had a history of pretreatments including TACE, RFA, resection, or some combination thereof. In the BCLC guidelines, TACE is commonly recommended for the treatment of intermediate-stage HCC. In contrast, due to technical advances in RFA and surgery in Japan, not a few patients undergo RFA or surgery as the first treatment for patients with intermediate-stage HCC [21, 22]. Japanese clinical practice guidelines for 
Kamo et al.: LT for Intermediate-Stage HCC

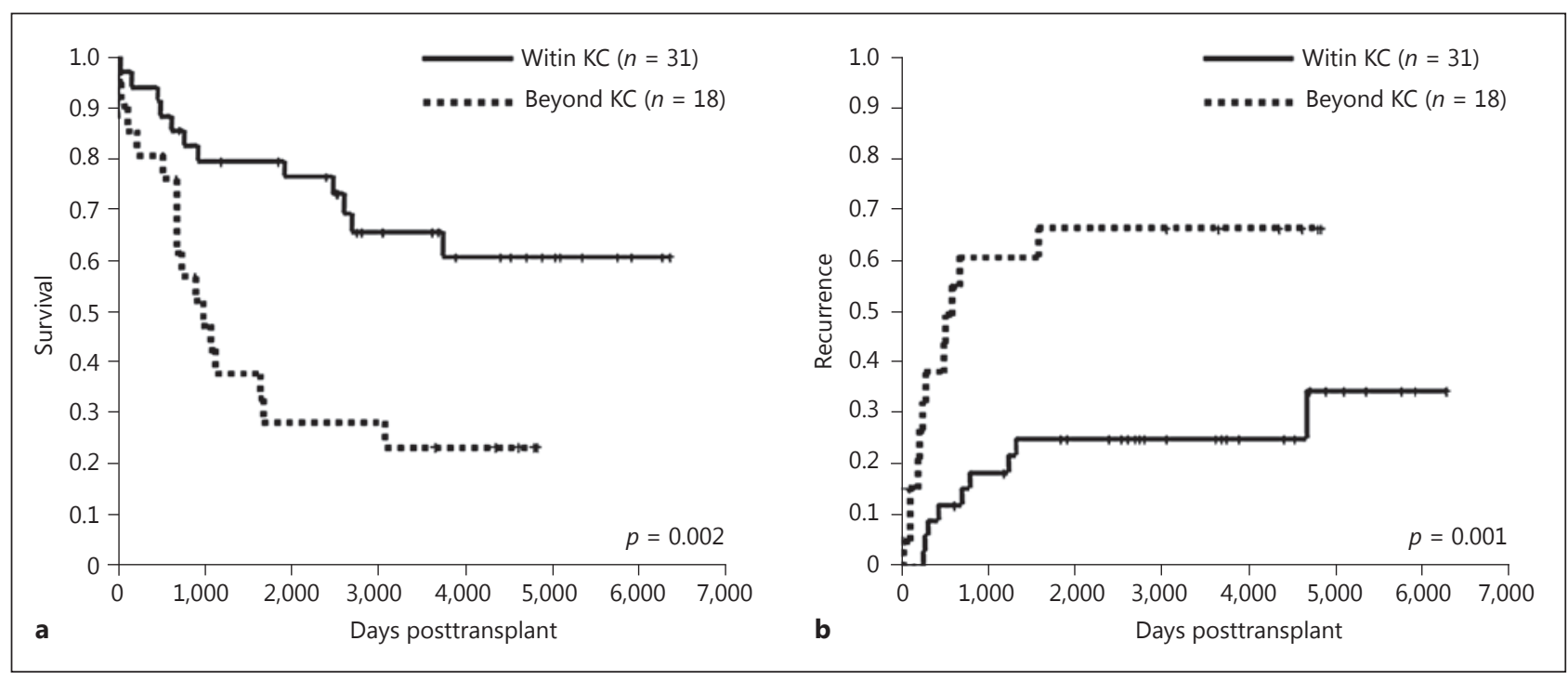

Fig. 6. Overall survival (a) and recurrence (b) rates of LT for intermediate-stage HCC with pretreatment within or beyond the KC. HCC, hepatocellular carcinoma; KC, Kyoto criteria; LT, liver transplantation.

HCC recommend various treatments including HR, RFA, and TACE for patients with ChildPugh classes A and B [7]. In the present study, therefore, Child-Pugh class A patients with pretreatment were significantly more frequent than those without pretreatment. If uncontrolled with these treatments, patients with HCC are referred to transplant centers, including our own, for LT as a second- or third-line treatment.

In the present study, patients with pretreatment had worse overall survival and recurrence-free survival rates compared with those without pretreatment, although there were no statistical differences. For this reason, the incidence of poorly differentiated HCC and microvascular invasion was higher in patients with pretreatment than in patients without pretreatment, although there were no statistical differences (Table 3). Therefore, tumor biology in patients with pretreatment could be more aggressive than that in patients without pretreatment. As the number of patients in this study was small, further investigations are needed to confirm the reason.

We reported that pretreatment of HCC was an independent risk factor for HCC recurrence after LT in all patients who underwent LT for HCC [20]. In the present study, however, overall survival and recurrence rates in patients with intermediate-stage HCC, especially in patients within the $\mathrm{KC}$, did not differ significantly between patients with and without pretreatment, again demonstrating the usefulness of the $\mathrm{KC}$ as expanded selection criteria. Patients with pretreatment within the $\mathrm{KC}$ also showed favorable outcomes compared with patients with pretreatment beyond the KC.

Some investigators have recently proposed the subclassifications of patients with intermediate-stage HCC, because intermediate-stage HCC includes various conditions of HCC and predicting survival rates is difficult [23-25]. Bolondi et al. [23] proposed a new subclassification using the MC and up-to-7 criteria. This method classified 4 substages of intermediate stage, B1-B4, showing recommended and alternative treatments for each substage. LT was recommended as an alternative treatment option for patients with a Child-Pugh score of 5-7 in substage B1 and a Child-Pugh score of 8-9 in substage B4. Yamakado et al. [24] divided patients undergoing chemoembolization based on the Child-Pugh score into 3 subgroups 
(B1-B3). They proposed LT for patients with a Child-Pugh score of 9 in substage B3. They showed that 2-year survival rates were $77.2 \%$ in 139 patients with 4 tumors measuring $\leq 7$ cm with a Child-Pugh class A (B1) ( $p<0.0001$ vs. B2), 59.5\% in the 178 patients other than B1 and B3 ( $p=0.0014$ vs. B3), and $16.7 \%$ in the 12 patients with a Child-Pugh score of 9 (B3). Furthermore, Kudo et al. [25] modified the subclassification proposed by Bolondi et al. by applying the Child-Pugh score for liver function and the MC and up-to-7 for tumor conditions. They recommended curative treatment such as resection, ablation, and superselective conventional TACE for stage B1 (beyond the MC and within up-to-7 with ChildPugh scores of 5-7), noncurative, palliative treatment such as drug-eluting beads TACE, hepatic arterial infusion chemotherapy, and sorafenib for stage B2 (beyond up-to-7 with Child-Pugh scores of 5-7), curative intent if tumors were within up-to-7 with Child-Pugh scores of 8-9 including LT, ablation, and superselective conventional TACE in stage B3-a, and only palliative treatment in stage B3-b (beyond up-to-7 with Child-Pugh scores of 8-9). The present study revealed that outcomes after LT are preferable for patients with intermediate-stage HCC if they meet the KC, regardless of pretreatments. This finding resembles the proposal from Kudo et al. regarding stage B3, although the KC are wider criteria than the up-to-7 criteria.

We previously reported outcomes of LT for small HCC [26]. If we recognize small HCC as early-stage HCC, 1-, 3-, and 5-year overall survival and recurrence rates within KC were $89 / 85 / 83 \%$ and $2 / 3 / 4 \%$, respectively. On the other hand, $1-, 3-$, and 5 -year overall survival and recurrence rates for patients with intermediate-stage HCC within KC were 94/80/80\% and $9 / 15 / 22 \%$, respectively. Although recurrence rates of patients with intermediate-stage HCC were higher than those of patients with early-stage HCC, overall survival rates of intermediate-stage HCC were almost similar to those of early-stage HCC. Taking the finding that intermediate-stage HCC is a more advanced cancer than early-stage HCC into consideration, outcomes after LT for intermediate-stage HCC would be favorable.

In the present cohort study, 8 out of 35 patients within the KC had recurrences. Three patients had intrahepatic and 5 patients had extrahepatic recurrences. On the other hand, 12 out of 21 patients beyond the KC had recurrences, which consisted of 3 intrahepatic and 9 extrahepatic recurrences. Patients beyond the KC seemed to be easier to have extrahepatic recurrence compared with patients within the $\mathrm{KC}$, although there was no statistical difference. All intrahepatic recurrences were considered to be metastatic. Compared with intrahepatic recurrence, extrahepatic recurrence might be hard to control, and immune checkpoint inhibitors could be applicable for such cases. However, LDLT and subsequent immune suppressive control could restrict the application of immune checkpoint inhibitors for extrahepatic or uncontrolled recurrence, although the effect of calcineurin inhibitor on immune checkpoint inhibitors is unclear. Therefore, we think it is important to use criteria with a low recurrence rate such as the KC.

Some limitations must be considered for this study. First, this study investigated a small cohort, especially for patients within the KC. A larger cohort study is required for validation of our results. Second, considering that data were collected retrospectively, the analysis could have limited identification of numerous confounding factors and led to some degree of selection bias. Third, this was a single-center study. Even if our institution were the largest LT center in Japan, a nationwide study would be desirable to confirm the present findings.

\section{Conclusion}

Outcomes after LT for intermediate-stage HCC are more favorable if patients meet our extended criteria. 
Kamo et al.: LT for Intermediate-Stage HCC

\section{Acknowledgements}

The authors would like to thank Ms. Mayumi Kawashima for her help with collecting the data for this study.

\section{Disclosure Statement}

The authors declare no conflicts of interest.

\section{References}

$>1$

$>2$

$>3$

$>4$

Harlan LC, Parsons HM, Wiggins CL, Stevens JL, Patt YZ: Treatment of hepatocellular carcinoma in the community: disparities in standard therapy. Liver Cancer 2015;4:70-83.

5 Kudo M, Trevisani F, Abou-Alfa GK, Rimassa L: Hepatocellular carcinoma: therapeutic guidelines and medical treatment. Liver Cancer 2017;6:16-26.

6 Llovet JM, Burroughs A, Bruix J: Hepatocellular carcinoma. Lancet 2003;362:1907-1917.

7 Kokudo N, Hasegawa K, Akahane M, Igaki H, Izumi N, Ichida T, Uemoto S, Kaneko S, Kawasaki S, Ku Y, Kudo M, Kubo S, Takayama T, Tateishi R, Fukuda T, Matsui O, Matsuyama Y, Murakami T, Arii S, Okazaki M, Makuuchi M: Evidence-based clinical practice guidelines for hepatocellular carcinoma: The Japan Society of Hepatology 2013 update (3rd JSH-HCC Guidelines). Hepatol Res 2015;45:123-127.

-8 Yao FY, Xiao L, Bass NM, Kerlan R, Ascher NL, Roberts JP: Liver transplantation for hepatocellular carcinoma: validation of the UCSF-expanded criteria based on preoperative imaging. Am J Transplant 2007;7:2587-2596.

-9 Mazzaferro V, Llovet JM, Miceli R, Bhoori S, Schiavo M, Mariani L, Camerini T, Roayaie S, Schwartz ME, Grazi GL, Adam R, Neuhaus P, Salizzoni M, Bruix J, Forner A, De Carlis L, Cillo U, Burroughs AK, Troisi R, Rossi M, Gerunda GE, Lerut J, Belghiti J, Boin I, Gugenheim J, Rochling F, Van Hoek B, Majno P; Metroticket Investigator Study Group: Predicting survival after liver transplantation in patients with hepatocellular carcinoma beyond the Milan criteria: a retrospective, exploratory analysis. Lancet Oncol 2009;10:35-43.

10 Sugawara Y, Tamura S, Makuuchi M: Living donor liver transplantation for hepatocellular carcinoma: Tokyo University series. Dig Dis 2007;25:310-312.

11 Toso C, Asthana S, Bigam DL, Shapiro AM, Kneteman NM: Reassessing selection criteria prior to liver transplantation for hepatocellular carcinoma utilizing the scientific registry of transplant recipients database. Hepatology 2009;49:832-838.

12 Zheng SS, Xu X, Wu J, Chen J, Wang WL, Zhang M, liang TB, Wu LM: Liver transplantation for hepatocellular carcinoma: Hangzhou experiences. Transplantation 2008;85:1726-1732.

-13 Takada Y, Ito T, Ueda M, Sakamoto S, Haga H, Maetani Y, Ogawa K, Ogura Y, Oike F, Egawa H, Uemoto S: Living donor liver transplantation for patients with HCC exceeding the Milan criteria: a proposal of expanded criteria. Dig Dis 2007;25:299-302.

14 Inomata Y, Uemoto S, Asonuma K, Egawa H: Right lobe graft in living donor liver transplantation. Transplantation 2000;69:258-264.

15 Ito T, Kiuchi T, Egawa H, Kaihara S, Oike F, Ogura Y, Fujimoto Y, Ogawa K, Tanaka K: Surgery-related morbidity in living donors of right-lobe liver graft: lessons from the first 200 cases. Transplantation 2003;76:158-163.

16 Morioka D, Egawa H, Kasahara M, Ito T, Haga H, Takada Y, Shimada H, Tanaka K: Outcomes of adult-to-adult living donor liver transplantation: a single institution's experience with 335 consecutive cases. Ann Surg 2007;245:315-325.

17 Inomata Y, Tanaka K, Egawa H, Uemoto S, Ozaki N, Okajima H, Satomura k, Kiuchi M, Yamaoka Y, Hashida T: The evolution of immunosuppression with FK 506 in pediatric living related liver transplantation. Transplantation 1996;61:247-252.

-18 Takada Y, Ueda M, Ito T, Sakamoto S, Haga H, Maetani Y, Ogawa K, Kasahara M, Oike F, Egawa H, Tanaka K: Living donor liver transplantation as a second-line therapeutic strategy for patients with hepatocellular carcinoma. Liver Transpl 2006;12:912-919.

19 Fukumitsu K, Hammad A, Kaido T, Ogawa K, Fujimoto Y, Mori A, Okajima H, Uemoto S: Validation of steroidfree immunosuppression regimen after liver transplantation. J Clin Gastroenterol Treat 2015;1:1-4.

20 Kaido T, Ogawa K, Mori A, Fujimoto Y, Ito T, Tomiyama K, Takada Y, Uemoto S: Usefulness of the Kyoto criteria as expanded selection criteria for liver transplantation for hepatocellular carcinoma. Surgery 2013;154: 1053-1060. 
21 Kariyama K, Wakuta A, Nishimura M, Kishida M, Oonishi A, Ohyama A, Nouso K, Kudo M: Percutaneous radiofrequency ablation for intermediate-stage hepatocellular carcinoma. Oncology 2015;89:19-26.

22 Tada T, Kumada T, Toyoda H, Tsuji K, Hiraoka A, Itobayashi E, Nouso K, Kariyama K, Ishikawa T, Hirooka M, Hiasa Y: Role of hepatic resection in patients with intermediate-stage hepatocellular carcinoma: a multicenter study from Japan. Cancer Sci 2017;108:1414-1420.

-23 Bolondi L, Burroughs A, Dufour JF, Galle PR, Mazzaferro V, Piscaglia F, Raoul JL, Sangro B: Heterogeneity of patients with intermediate (BCLC B) hepatocellular carcinoma: proposal for a subclassification to facilitate treatment decision. Semin Liver Dis 2012;32:348-359.

-24 Yamakado K, Miyayama S, Hirota S, Mizunuma K, Nakamura K, Inaba Y, Yamamoto S, Matsuo K, Nishida N, Aramaki T, Anai H, Kora S, Oikawa S, Watanabe K, Yasumoto T, Furuichi K, Yamaguchi M: Prognosis of patients with intermediate stage hepatocellular carcinomas based on the Child-Pugh score: subclassifying the intermediate stage (Barcelona Clinic Liver Cancer stage B). Jpn J Radiol 2014;32:644-649.

25 Kudo M, Arizumi T, Ueshima K, Sakurai T, Kitano M, Nishida N: Subclassification of BCLC B stage hepatocellular carcinoma and treatment strategies: proposal of modified Bolondi's subclassification (Kinki Criteria) for patients with intermediate stage hepatocellular carcinoma. Dig Dis 2015;33:751-758.

26 Kamo N, Kaido T, Yagi S, Okajima H, Uemoto S: Liver transplantation for small hepatocellular carcinoma. Hepatobiliary Surg Nutr 2016;5:391-398. 